\title{
The performance of AGATA: From the LNL demonstrator to the GANIL setup
}

\author{
Caterina Michelagnoli
}

GANIL CEA/DSM-CNRS/IN2P3, Caen, France

\begin{abstract}
The performance of the Advanced-GAmma-Tracking-Array AGATA is demonstrated through selected examples from the different recent experimental campaigns.
\end{abstract}

\section{Introduction}

In order to perform nuclear structure studies in extreme conditions of neutron/proton asymmetry and angular momentum, in the last decade, the challenge of the realization of the gamma-ray spectroscopy dream has been taken on by two international collaborations, AGATA (Advanced-GAmmaTracking-Array) [1] in Europe and GRETA (Gamma-Ray Energy Tracking Array) in the US [2]. The dream consists in covering the whole $4 \pi$ solid angle by germanium material and track the path of the gamma rays inside the detector medium, corresponding to maximum efficiency and "infinite" position resolution, in addition to an excellent energy resolution.

On this purpose, the digitally recorded waveforms from highlysegmented HPGe detectors are treated with Pulse-Shape-Analysis (PSA) techniques in order to extract the position of the interaction points in the detector with a $4 \mathrm{~mm}$ position resolution (at $1 \mathrm{MeV})[3,4]$. These interaction points (hits) are grouped in events on the basis of the timestamp. Different gamma rays in the same event and their ordered path are reconstructed via tracking algorithms. An efficiency four times larger than standard arrays 
(also for high gamma-ray multiplicities) is expected, together with the capability to stand high count-rate (up to hundreds of $\mathrm{kHz}$ ) thanks to the use of digital electronics. For the first time an almost continuous angular distribution of gamma interaction points will be available, allowing for a "perfect" Doppler correction and new degree of sensitivity in the determination of nuclear structure observables.

This contribution focuses on the AGATA array. It run its Demonstrator phase in 2009-2010 [5] and first physics campaign with stable beams at the Legnaro National Laboratories, consisting mainly in the spectroscopy and lifetime measurements in neutron-rich nuclei produced in deep-inelastic reactions at the PRISMA magnetic spectrometer. It then moved to GSI for the PreSpec campaign at the FRS separator and used for the first measurements with fast moving radioactive ion beams (beta up to $70 \%$ ). It is now running its first experimental campaign at GANIL (France) coupled to the large acceptance magnetic spectrometer VAMOS [6]. Details on the AGATA project can be found in the reference paper [1]. It the following, the focus will be on particular aspects, that bring to new physics opportunities already with a sub-system of the whole array.

\section{High count-rate capabilities}

The use of digital electronics to preprocess the detector preamplifier signals allow to preserve a good efficiency and energy resolution even for count-rates larger than $10 \mathrm{kHz}$ (typical limitation when using analogue electronics). In Fig. 1 an example of the output of the detector preamplifier on the core contact of an AGATA detector in response to a count rate of $30 \mathrm{kHz}$ is shown (in red), together with the corresponding amplitudes reconstructed via the trapezoidal filter, corresponding to two different risetimes of the trapezoid (in blue and black). The proper choice of the risetime (and of the filter parameters in general), together with the baseline restoration, allow for extracting the amplitudes from the signals and avoiding pileup. A detailed study of the efficiency and resolution of an AGATA detector as a function of the count rate is reported in [7].

\section{Correction for neutron damage}

The operation of the germanium detectors in position-sensitive mode allows for the correction for the effects of the damage of the germanium crystalline 

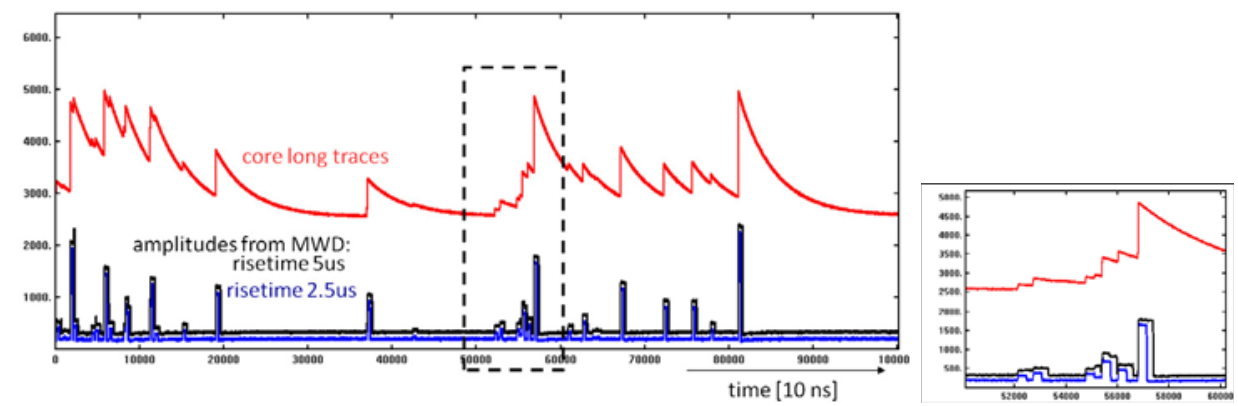

Figure 1: Response of the core preamplifier of an AGATA HPGe detector to an average $30 \mathrm{kHz}$ count rate, during an in-beam experiment (in red). The amplitudes extracted from the signals when using a trapezoidal filter with two different risetimes are reported in black and blue. In the right panel, a zoom of the region indicated by the dashed rectangle is reported. The shorter risetime acts as a pile-up rejector.

structure due to the interaction of fast neutrons. Such an effect results in an incomplete charge collection causing a worsening of the energy resolution.

Since the charge loss due to neutron damage is proportional to the length of the path to the electrodes, the interaction points resulting from PSA can be used together with a modeling of charge trapping to correct for such an effect [8]. In Fig. 2 the energy spectra corresponding to the $1.3 \mathrm{MeV}$ line $\left({ }^{60} \mathrm{Co}\right.$ calibration source) before and after the correction are compared. This procedure is available and used for the detectors that suffers from neutron damage and applied in the data flow (or offline processing) after the PSA, before the data are passed to the global level stage, where event building and tracking are performed.

\section{Some benefits from position resolution}

The position resolution of AGATA can be used for the determination of nuclear structure observables with a new degree of sensitivity, giving access to new physics opportunities. Few selected examples from the Legnaro and GANIL campaigns will be given in the following. 


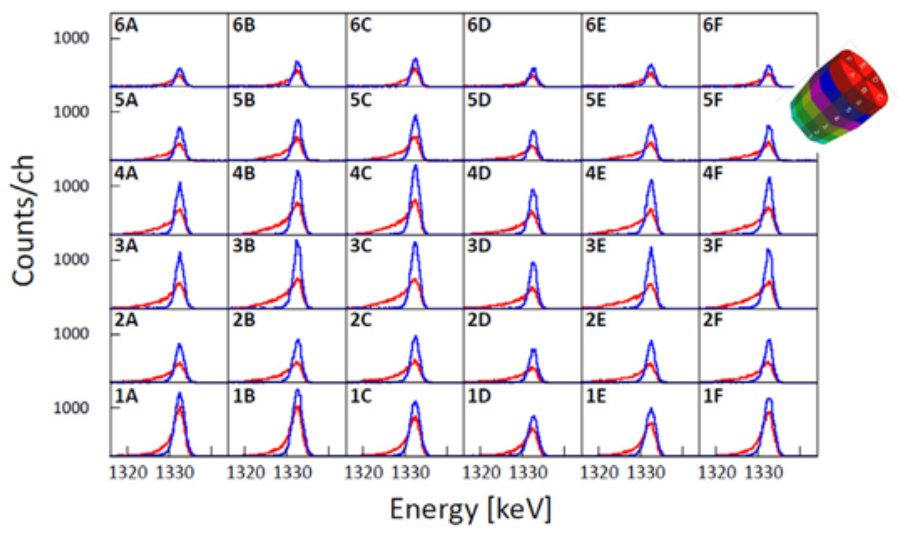

Figure 2: Segment spectra corresponding to the $1.3 \mathrm{MeV}$ line $\left({ }^{60} \mathrm{Co}\right.$ calibration source) -see schematic representation of an AGATA detector in the inset for segment labeling. The left tail consequent to inefficiency in charge collection due to neutron damage is evident in the spectra in red (no correction applied) and it is significantly reduced in the spectra in blue, obtained after correction for neutron damage (resolution for the sum of all segments from $5.9 \mathrm{keV}$ to $2.9 \mathrm{keV}$ after correction. The data are taken during the Legnaro campaign (picture taken from [9]).

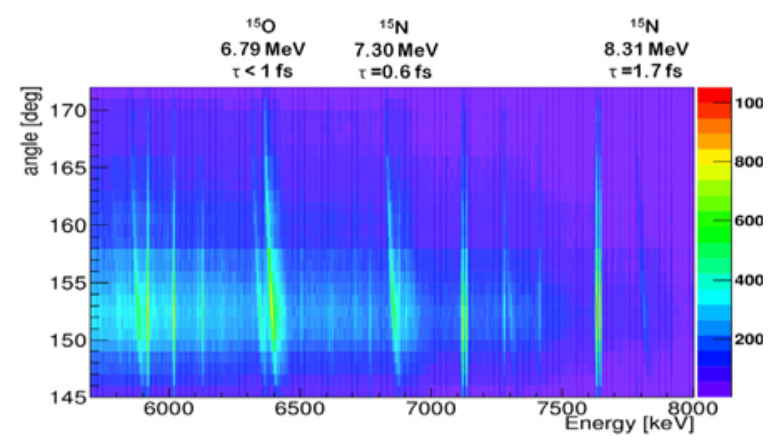

Figure 3: Tracked gamma-ray energy sorted according to the angle of the reconstructed first interaction point, from the experiment for the measurement of the lifetime of the $6.79 \mathrm{MeV}$ state in ${ }^{15} \mathrm{O}$. The "straight" lines correspond to the emission at rest by a radioactive source (present while beam-on-target for monitoring of gain instabilities). The tilted broad lines correspond to gamma rays emitted from short $(\approx \mathrm{fs})$ lived states. The lifetimes are obtained with Doppler Shift Attenuation technique over an almost continuous distribution of angles. 


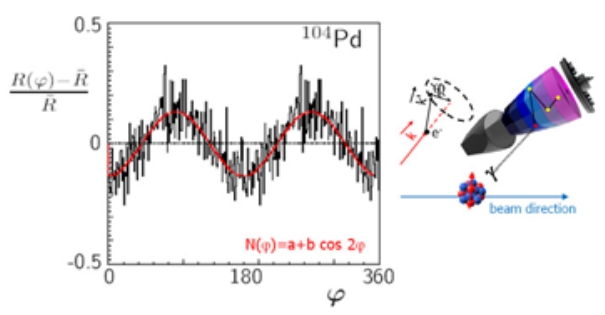

Figure 4: (taken from [11]) Experimental asymmetry as a function of the azimuthal angle of Compton scattering measured for the deexcitation of the first $2^{+}$state in ${ }^{104} \mathrm{Pd}$ populated by Coulomb excitation. The gamma linear polarization is obtained with a fit to the experimental data [11].

\subsection{Sub-femstosecond lifetime of interest in nuclear astro- physics}

The position resolution of AGATA can be used to measure nuclear level lifetimes via Doppler Shift techniques exploiting a continuous distribution of angles. It is well known that especially in the case of the Doppler Shift Attenuation Method (DSAM) the contribution of the detector response function, namely the finite angular coverage of the detectors, is one of the main factors that limits the sensitivity. A new sub-femtosecond upper limit has been recently obtained for the $6.79 \mathrm{MeV}$ state in ${ }^{15} \mathrm{O}[10]$. The experiment was performed during the Demonstrator campaign in Legnaro. The reaction of ${ }^{14} \mathrm{~N}$ beam on a ${ }^{2} \mathrm{H}$ target has been used to populate the state of interest. The effect of fs lifetimes can be observed by analysing the line-shape of the gamma rays, emitted by the reaction products slowing down in a gold layer, as a function of the observation angle. In Fig. 3 the energy of the reconstructed gamma rays sorted according to the angle of the first interaction point is reported with an unprecedented angular resolution of 2 degrees (see figure caption for details).

\subsection{AGATA as a Compton polarimeter}

The second point of interaction of a gamma ray in its attenuation path in the germanium medium can be used to determine the linear polarization of gamma rays emitted by aligned nuclear states. The asymmetry in the distribution of the Compton scattered gamma rays as a function of the azimuthal angle can be used to determine the linear polarization. A test of AGATA modules as Compton polarimeters has been performed in the Legnaro campaign [11], using the Coulomb excitation of the first excited 


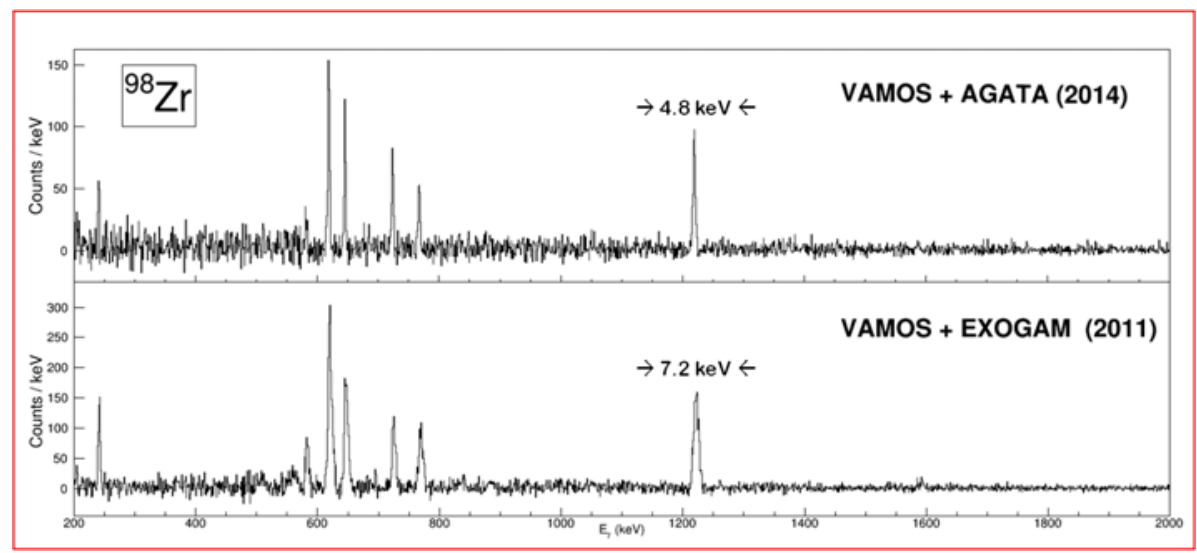

Figure 5: Gamma-ray spectra corresponding to the ${ }^{98} \mathrm{Zr}$ nucleus produced in the fission of a ${ }^{238} \mathrm{U}$ beam on a ${ }^{9} \mathrm{Be}$ target at $6.6 \mathrm{MeV} / \mathrm{u}$ and identified in the magnetic spectrometer VAMOS. The comparison of performance in gamma-ray detection between the EXOGAM (lower panel, data taken from [13]) and AGATA (upper panel, data taken from December 2014 commissioning runs) arrays is shown. The FWHM at $1.2 \mathrm{MeV}$ is reported.

states in ${ }^{104,106} \mathrm{Pd}$ targets by a ${ }^{12} \mathrm{C}$ beam. As evident from Fig. 4 (taken from [11]), with respect to standard clover detection system, this asymmetry can be analysed as a continuous function of the angle. This results in a larger analysing power, that allows for the measurement of gamma linear polarization in cases of weaker nuclear alignments or, in general, for rarer phenomena. This is the case of the study of the photon entanglement through the measurement of the relative polarization of the $511 \mathrm{keV}$ gamma rays emitted by the singlet positronium [12].

\subsection{Doppler correction capabilities}

In Fig. 5 the advantages of using PSA and tracking techniques for the detection of gamma rays with respect to traditional arrays are shown, by using the comparison of spectra obtained in the same experimental conditions, exception made for the gamma array. The ${ }^{98} \mathrm{Zr}$ nucleus was populated in the fission process in inverse kinematics of ${ }^{238} \mathrm{U}$ beam on a ${ }^{9} \mathrm{Be}$ target at 6.6 MeV/u. The same reaction has been recently used in GANIL for the study of isotopically-identified neutron-rich fission fragments at the VAMOS ++ magnetic spectrometer [13]. Here a spectrum obtained from those data is used for comparison. The gain of almost a factor of two in energy resolution is evident. 


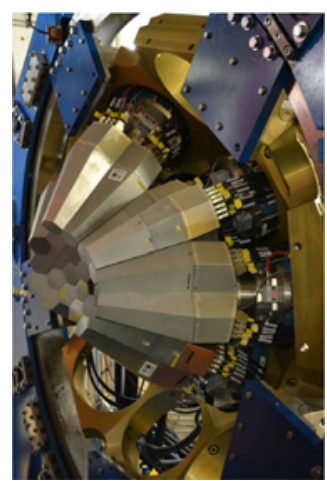

(a) AGATA at GANIL (eight triple clusters).

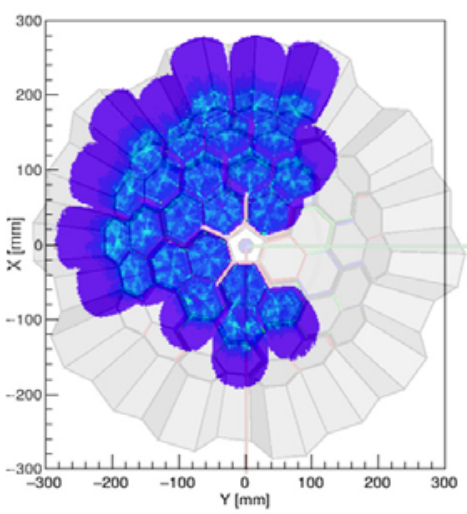

(b) Hit pattern from 24 crystals.

Figure 6: The AGATA array installed for the first campaign at the VAMOS spectrometer at GANIL (spring/summer 2015). Eight triple clusters are mounted at backward angles with respect to the axis of the VAMOS spectrometer. The corresponding hits reconstructed via PSA are also shown (in the XY plane) together with the simulation of the $1 \pi$ array foreseen for the forthcoming experimental campaign at GANIL.

\section{The AGATA+VAMOS campaign at GANIL}

The commissioning runs in GANIL took place in November-December 2014 and the Doppler correction capabilities and physics correlations between the gamma and magnetic spectrometers were verified and tested for different range of velocities and $\mathrm{A}$ and $\mathrm{Z}$ of the emitting nuclei [14].

Nowadays 8 triple clusters of the AGATA array are available, Fig. 6. The AGATA array positioned at backward angles with respect to the beam line coupled to the magnetic spectrometer VAMOS ++ were used in the first campaign with stable beams at GANIL, in March-July 2015. The sensitivity of this unique combination was used to measure lifetimes and other spectroscopic properties of neutron rich nuclei produced by fission and deepinelastic reactions in different region of the nuclear chart.

\section{References}

[1] S. Akkoyun et al., Nuclear Instruments and Methods in Physics Research Section A 668, 26 (2012) 
[2] I. Lee et al., Nuclear Physics A 746, 255 (2004)

[3] F. Recchia et al., Nuclear Instruments and Methods in Physics Research Section A 604, 555 (2009)

[4] P.A. Söderstrom et al., Nuclear Instruments and Methods in Physics Research Section A 638, 96 (2011)

[5] A. Gadea et al., Nuclear Instruments and Methods in Physics Research Section A 654(1), 88 (2011)

[6] M. Rejmund et al., Nuclear Instruments and Methods in Physics Research Section A 646(1), 184 (2011)

[7] F. Recchia et al., LNL Annual Report 2010 p. 60 (2011)

[8] B. Bruyneel et al., The European Physical Journal A 49 (2013)

[9] C. Michelagnoli, Ph.D. thesis, University of Padova (2013)

[10] C. Michelagnoli et al., submitted to PRL

[11] P. Bizzeti et al., The European Physical Journal A 51(4) (2015)

[12] P. Bizzeti et al., private communication

[13] A. Navin et al., Physics Letters B 728, 136 (2014), ISSN 0370-2693

[14] C. Michelagnoli et al., in preparation 